\title{
AGGREGATED LINE MODELING FOR SIMULATION AND OPTIMIZATION OF MANUFACTURING SYSTEMS
}

\author{
Leif Pehrsson \\ Marcus Frantzén \\ Tehseen Aslam \\ Amos H.C. Ng \\ University of Skövde \\ SE-541 28 Skövde, SWEDEN
}

\begin{abstract}
In conceptual analysis of higher level manufacturing systems, for instance when the constraint on system level is sought, it may not be very practical to use detailed simulation models. Developing detailed models on supply chain level or plant wide level may be very time consuming and might also be computationally costly to execute, especially if optimization techniques are to be applied. Aggregation techniques, simplifying a detailed system into fewer objects, can be an effective method to reduce the required computational resources and to shorten the development time. An aggregated model can be used to identify the main system constraints, dimensioning inter-line buffers, and focus development activities on the critical issues from a system performance perspective. In this paper a novel line aggregation technique suitable for manufacturing systems optimization is proposed, analyzed and tested in order to establish a proof of concept while demonstrating the potential of the technique.
\end{abstract}

\section{INTRODUCTION}

For many years production lines have successfully been modeled and simulated using Discrete Event Simulation (DES) techniques. When combining DES with optimization technology, and Multi Objective Optimization (MOO) in particular, valuable decision support information can be retrieved from the models (Pehrsson and $\mathrm{Ng}$ 2011). Furthermore, the addition of knowledge extraction techniques has proven to produce very useful information for supporting decision-making (Dudas et al. 2013; $\mathrm{Ng}$ et al. 2012). The knowledge extraction process used is mainly derived from the concept of "Innovization", explained as innovation through optimization (Deb and Srinivasan 2006). The application of these methods has mostly been conducted on production line level and has generated very successful results. Some applications on higher level systems, such as complete factories and supply chains has been done using mathematical models and methods (Aslam et al. 2011). But since most of these mathematical models are deterministic, like system dynamics models, using them for modeling stochastic systems can be misleading (Madan et al. 2005). Until recently, DES that models system stochastic behaviors has not been applied to the same extent on higher manufacturing systems level (Pehrsson et al. 2014; Beamon 1998; Hung et al. 2006). Sometimes, the mathematical methods are considered less accurate and might be more difficult to understand than the DES-based methods (Morecroft 2007). The DES models, on the other hand, have some significant drawbacks as they tend to be very time consuming, requiring detailed modelling when considering analysis and optimization of complete factories, and as they often require extensive computing resources and long execution times in such situations. Consequently, DES methods are generally considered to be less suited for higher level manufacturing system analysis. One way of 


\section{Pehrsson, Frantzén, Aslam, and Ng}

coping with the drawbacks and improve the applicability of DES methods on high level manufacturing systems would be to use aggregated DES modelling techniques for the replication of production lines. This paper proposes and explores a new DES aggregation technique for production lines, where the main objectives are to present a generally applicable aggregation technique that can be applied in conceptual development of manufacturing systems and takes into account the stochastic behaviors of the aggregated systems. The models created by the new aggregation technique should be less computational expensive compared to detailed model in order to be suitable for optimizations. As concluded in (Madan et al. 2005), stochastics should not be neglected regardless of the model fidelity selected.

Through an application study connected to a real world factory development project, an early proof of concept is achieved. In summary the objectives for this paper is to propose a technique for aggregated DES production line modeling fulfilling the following objectives: (1) suitable for use in high level systems, (2) with few commonly applicable input parameters, (3) suitable for conceptual modeling, optimization, and knowledge extraction, and (4) considering variation in output throughput (pace), input throughput, lead time and WIP. The remaining part of this paper is organized in three main sections, beginning with aggregated modeling principles, including the proposal of the new aggregation technique with an analysis of its' key properties, then an application study is presented, and finally the conclusions are summarized together with recommendations for future work.

\section{AGGREGATED MODELING PRINCIPLES}

Manufacturing systems can be studied and analyzed from various levels as pointed out by Rooda and Vervoort (2007). They distinguish between the four levels (1) machine level, (2) the workstation level, (3) the sub-network level, and (4) the network level. Additionally other levels could be added such as the process level describing e.g. the cutting process in machining, and the supply chain level including separate factories, transports, and buffers in a complete networked system. An effective way of analyzing complex manufacturing systems in various abstraction levels is to use modeling and simulations (Chwif, Barretto and Paul 2000). One of the most preferred tools among industrial practitioners for analyzing complex systems is DES (Madan et al. 2000). However, DES models do often require long execution times when compared to mathematical models which might be a limiting factor when simulation is connected to optimization, since inherently such procedures require a large number of simulation runs to produce the desired results. The model complexity is depending on the scope and the level of details required in a simulation model (Chwif, Barretto, and Paul 2000). The complexity can be reduced through aggregation or abstraction of the models whilst preserving the validity of the model in relation to the scope of the simulation (Frantz 1995). The opportunities to extend the applicability of DES methods to the network level and the supply chain level was investigated and a number of aggregation techniques were presented and analyzed by Pehrsson et al. (2014). The paper presented a strong indication that such models could produce useful results for supporting decision making on system level. However, one of the conclusions made by the authors was that the modeling techniques required further refinement and validation. Two of the issues pointed out by the authors were the statistical analysis required to populate the model and the difficulty to balance the input cycle time. Hence, the method is not well suited for conceptual analysis with generalized data assumptions. Additionally variation on a line's input side is not modeled.

\subsection{A new DES Aggregation technique}

In this section, a new approach for aggregating a complex system into a simplified DES model, based on a few standard DES objects, is proposed and described. According to Banks et al., 2010, Discrete-event system simulation, using the event scheduling/time advance algorithm, is the modeling of systems in which the state variable changes only at a discrete set of points in time. Consequently such models are analyzed numerically rather than analytically and deductively. The aggregated models were created and tested in the two independent DES software packages FACTS Analyzer (Ng et al. 2011) and Plant 


\section{Pehrsson, Frantzén, Aslam, and Ng}

Simulation with equal results. The type of data known or estimated during conceptual assumptions was discussed with industry partners in the search of suitable input parameters and viable aggregation techniques for conceptual modeling of production lines. There was no interest in deriving statistical data from existing lines, and preferred type of parameters were processing time, availability, WIP, lead time and throughput. The desired output parameters for network level analysis are throughput, WIP, and lead time.

Based on observations from earlier studies and the input from the industry partners a new strategy for aggregation of production lines is being proposed in an attempt to address several of the identified issues with the previous techniques by: (1) Considering input variation, (2) balancing the line WIP towards an expected average, and (3) simplifying the required inputs to more generalized parameters.

It is not an easy task to simultaneously model the variation in lead time, input variation, and output variation. Just adding input variation together with output variation as observed from real world production lines was tested and it very soon became apparent that the input variation and output variation created statistical interference degrading the throughput of the modeled line. Even though it might be possible to correct this through a statistical or mathematical analysis of the interference, a more elegant solution was sought. An alternative way of managing the WIP and the lead time would be to control the amount of products allowed to enter the line. In the literature, there are control theory based methods for the dynamic regulation of WIP in a production line, e.g. (Duffie and Shi 2009). Nevertheless, the challenge here is to both control the WIP in the line as well as modeling its stochastics. A new concept was introduced to keep the average amount of containers representing the difference between the maximum WIP and the average WIP outside the line. That kind of functionality can be realized by the addition of a pallet system and a WIP control storage, as shown in Figure 1.

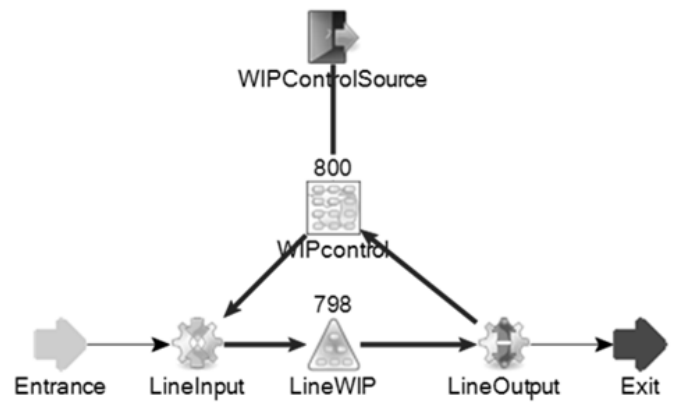

Figure 1: Proposed aggregation technique line model in FACTS Analyzer.

The standard DES objects used in the aggregated DES model are a source providing containers into the pallet system, a store in FACTS Analyzer or a parallel proc. in Plant Simulation was used as WIP control, an assembly used as line input, a buffer as Line WIP, and a disassembly as line output. By delaying pallets through the WIP control storage, the amount of products in the line will be restricted. A constant delay time would transfer the variation in the output object more or less instantaneously, be it delayed, to the input object. A more natural behavior can be obtained by adding variation to the delay time. In this case an exponential variation makes sense since it is a memoryless distribution (Banks et al. 2010) with a mean value as an input, often used for failure times and repair times in manufacturing system analysis (Curry and Feldman 2011). The average delay time can be determined by calculating the average throughput $(\mathrm{TH})$ of the system and the desired average amount of containers to be kept outside the system. The average delay time can then be used as the mean value in the exponential delay time distribution, implemented through the processing time in the WIP control object.

The delay time can be determined by an expression derived from Little's Law (Little, 1961) in a commonly used variant for manufacturing systems (Hopp and Spearman 2000) shown in Equation (1): 


$$
T H=\frac{W I P}{C T}
$$

Where $T H=$ Throughput (arrival rate), $W I P=$ Work In Progress, and $C T=$ the cycle time of the complete system, also defined as the expected time spent in the system, or the lead time $(L T)$ through the system.

The lead time spent in the system is then (2):

$$
L T=\frac{W I P}{T H}
$$

The average amount of WIP to reside in the WIP container is the difference between the maximum amount of WIP in the line and the average amount of WIP in the line (3):

$$
W I P_{c a}=W I P_{l m}-W I P_{l a}
$$

Where $W I P_{c a}=$ Average WIP in the WIP control object, $W I P_{l m}=$ Maximum WIP in the line, and $W I P_{l a}=$ the average WIP in the line.

The average throughput (per second) of the system, when running under ideal conditions, that is the line is never starved or blocked, can be determined by the processing time and the availability (4):

$$
T H_{l a}=\frac{1}{\alpha_{l a}} \cdot \beta_{l}=\frac{\beta_{l}}{\alpha_{l a}}
$$

Where $T H_{l a}=$ Throughput of the line, $\alpha_{l a}=$ the average processing time of the line, and $\beta_{l}=$ The availability of the line.

The mean value for the delay function in the WIP control object is then (5):

$$
L T_{d}=\frac{W I P_{l m}-W I P_{l a}}{T H_{l a}}=\frac{W I P_{l m}-W I P_{l a}}{\frac{\beta_{l}}{\alpha_{l a}}}=\frac{\left(W I P_{l m}-W I P_{l a}\right) \cdot \alpha_{l a}}{\beta_{l}}
$$

Where $L T_{d}=$ The lead time (delay) through the WIP control object.

The line model can be completed by setting the desired processing time, availability, and MTTR in the line output object. The line WIP object is simply a buffer object with a capacity representing the maximum WIP level that would be observed in a real world system minus 2 in order to compensate for the input and output objects. A first in first out (FIFO) buffer is used to represent a FIFO line and a storage object can be used to represent a non FIFO line. The processing time of the line WIP object represents the minimum lead time through the system after subtraction of the processing times in the line input and the line output. The line input object is an assembly operation since the incoming product will be assembled to the WIP container through the line WIP buffer and then disassembled at the line output. The line input object will use the same processing time as the line output object. 


\section{Pehrsson, Frantzén, Aslam, and Ng}

One concern with the proposed modeling technique is that the output and input are linked through the WIP control delay loop and longer disturbances with low frequency occurring in the beginning of the line might not be correctly modeled. This will primarily be an issue when dealing with larger lines that have comparably long lead times. In such cases some additional variation can be added through availability and MTTR in the line input object as long as statistical interference with the line output variation is avoided. Hence, additional input variation can be added as long as the throughput remain unaffected for a line running under ideal conditions. A choice has to be made on where to connect the WIP control source feeding the model with the right amount of WIP containers. If connected directly to the line input there will be no delay time at the start of the simulation and there will be no probability for long delay times stopping the line input. Connecting the WIP control source to the WIP control will put delay time according to the selected delay function on each container before entering the line. The latter behavior was selected in order to have the variation included already from the start of simulations. The influence from the selected WIP control source strategy can however be eliminated if taken into account when setting the warm up time for a simulation.

\subsection{Required input and how to populate the aggregated model with data}

In contrast to the vast number of inputs required for detailed DES models, the inputs required to describe an aggregated DES production line model can be simplified into a few logical parameters with the proposed technique, namely: Processing time, Availability, Mean Time To Repair (MTTR), Maximum WIP, Average WIP, Minimum lead time.

The input parameters are introduced into the aggregated model as follows. Processing time is used to set the pace of the system when running without disturbances. It is an input to the line output object, the line input object and as a part of the calculation of the delay time through the WIP control. The availability is used to invoke the right amount of variability in the line output object and it is also used when calculating the WIP control delay time. MTTR is an input to the line output object and controls the variation pattern in terms of the average length of the disturbances. Maximum WIP is controlling the maximum amount of products allowed into the line and is an input to the WIP container source, the WIP control capacity, the WIP control delay calculation, and the line WIP capacity where it is reduced by 2 in order to compensate for the line input and line output objects WIP capacity of one each. The average WIP is input to the WIP control delay time calculation and the minimum lead time is used as the processing time in the line WIP object after compensation for the two processing times in the input and output objects, serving as the minimum time a product must spend in the line.

\subsection{Analysis of the Aggregated Modeling technique}

The new DES aggregation technique is mainly intended to be used for rapid modeling and optimization of higher level conceptual manufacturing systems. The idea is to use a simplified DES model with only a few input parameters, i.e. Processing time, Availability, MTTR, Maximum WIP, Average WIP, Minimum lead time, that can be estimated during the development of conceptual systems. Data from a component production line (CL1) presented and analyzed by Pehrsson et al. (2014) was used to test and analyze the new aggregation technique, shown in Figure 1 with such parameters. In these analyses, the processing time has been generalized to be constant in order to meet the objectives with few input parameters. However, processing time variation is an available option in the modeling environment. The main variation in the model is generated in the line output object through the availability and the MTTR modeling. The availability is simply the percentage of time when the line is producing at the processing time and the MTTR is the average length of the line stops. Behind that data, the mean time to failure, i.e. the time from the end of one failure to the start of the next one, uses an exponential distribution and the MTTR uses an Erlang distribution with k-value 2. Generally the exponential distribution can be used to model the time between independent events and the Erlang distribution to model the sum of several exponentially distributed processes (Banks et al. 2010). Furthermore, the WIP control storage object, use 
the exponential distribution for the processing time. As a result of delaying WIP containers through the WIP control, realistic input variation is generated through the transferred output variation in combination with the addition of the exponential variation of the WIP control delay time. At the same time the WIP and lead time are controlled to replicate the modeled line.

In order to investigate if the proposed aggregation technique offers the potential to produce reasonably good results when replicating real world production lines, a comparison of two output measures, finish times and lead time, was carried out. The finish times are simply the times when each work piece is finished in the last operation. The finish times is the collective product of disturbances and other delays, e.g. machine failures and setup times, in the production system. The output data generated from the previous (Pehrsson et al. 2014) and the new aggregation techniques are compared with the realworld data for finish times in Figure 2 (left) and the lead time variation is shown in Figure 2 (right). The finish times show a fairly close resemblance between the real-world data and the simulation model data. Furthermore, the simulation model seems to generally capture the lead-time variation of the real-world production line when considering the variation from one day to another. There are, however, a few high level spikes and a few short lead-times are not fully captured with the aggregated model. The most essential output measures of a model are in general the mean values of WIP, lead time and throughput, and they are often used for building deterministic models of larger systems (Aslam, 2013). A comparison of the mean values for throughput (TH) in jobs per hour (JPH) and lead-time (LT) in seconds (s), from the simulation model and the production line are shown in Table 1. The fourth column in Table 1 shows a low result deviation, when comparing the simulation model's mean value to the mean value of the production line. Consequently, the new aggregation strategy is considered being able to capture the main behavior of a whole system with only a few DES objects, based on generalized input data that can be estimated during conceptual development of manufacturing systems.
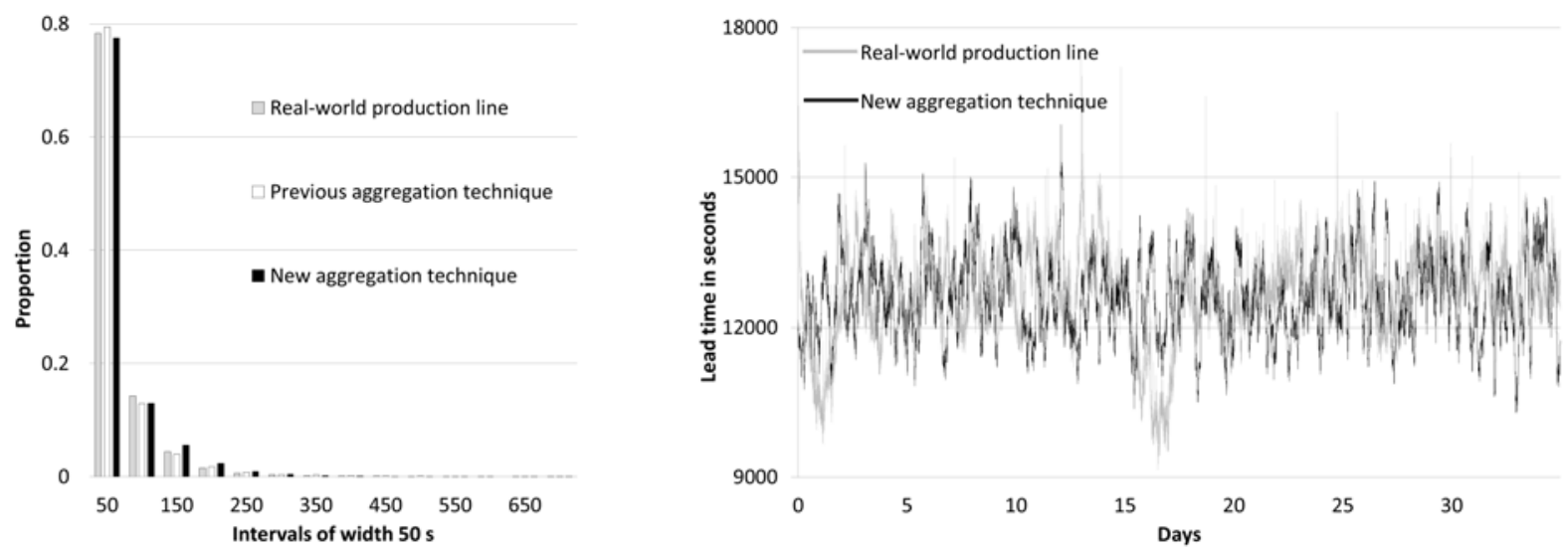

Figure 2: Left: Histogram of finish times. Right: Lead-time variation comparison.

Table 1: Mean values comparison.

\begin{tabular}{|l|l|l|l|}
\hline Output & Aggregated model & Real-world production line & Result deviation \\
\hline TH (JPH) & 62.67 & 62.68 & $\sim-0.02 \%$ \\
\hline LT (s) & 12662.43 & 12604.04 & $\sim+0.46 \%$ \\
\hline
\end{tabular}

\subsection{Aggregated modeling conclusions}

The main purpose of the aggregated modelling is to sufficiently replicate the overall behavior of a production line in order to perform studies on a larger scale manufacturing system and on average the 
most important factors can be modeled very accurately. Many of the existing procedures for supply chain analysis does not fully take variation into account and just having a fairly good estimation of the variability in a production line is considered to be enough. The proposed aggregation method takes variability into account from several perspectives. The output variation can be modelled more or less spot on with the system being replicated. When it comes to lead time, maybe the most difficult part to replicate, the results are fairly close to the real-world production line. Input variation is included as a combination of distributed variation from the line output through the WIP control loop and the added variation in the WIP control loop. The opportunity to add some more variation in the line input makes sure that reasonably accurate estimations can be done.

\section{APPLICATION STUDY}

The new aggregation technique presented in this paper is supposed to enable conceptual analysis of manufacturing systems and supply chains with aggregated lines based on a few assumptions about the line's performance expressed in commonly used parameters for manufacturing system development. A real world conceptual development project within automotive component production was closely monitored in order to make an application study aiming for a proof of concept. The conceptual development manager at the company was closely involved during the modelling, providing the assumptions about each lines' performance. The model was built in FACTS Analyzer (Ng et al. 2011), a software with the capabilities of handling conceptual models, possibility to create custom objects, combined with extensive simulation, optimization, and analysis functionality. In order to protect sensitive information from the company some of the data had to be concealed, however the relations in the data have been preserved. The conceptual factory analyzed comprises nine machining lines, six assembly lines, and a shipping department, as shown in Figure 3.

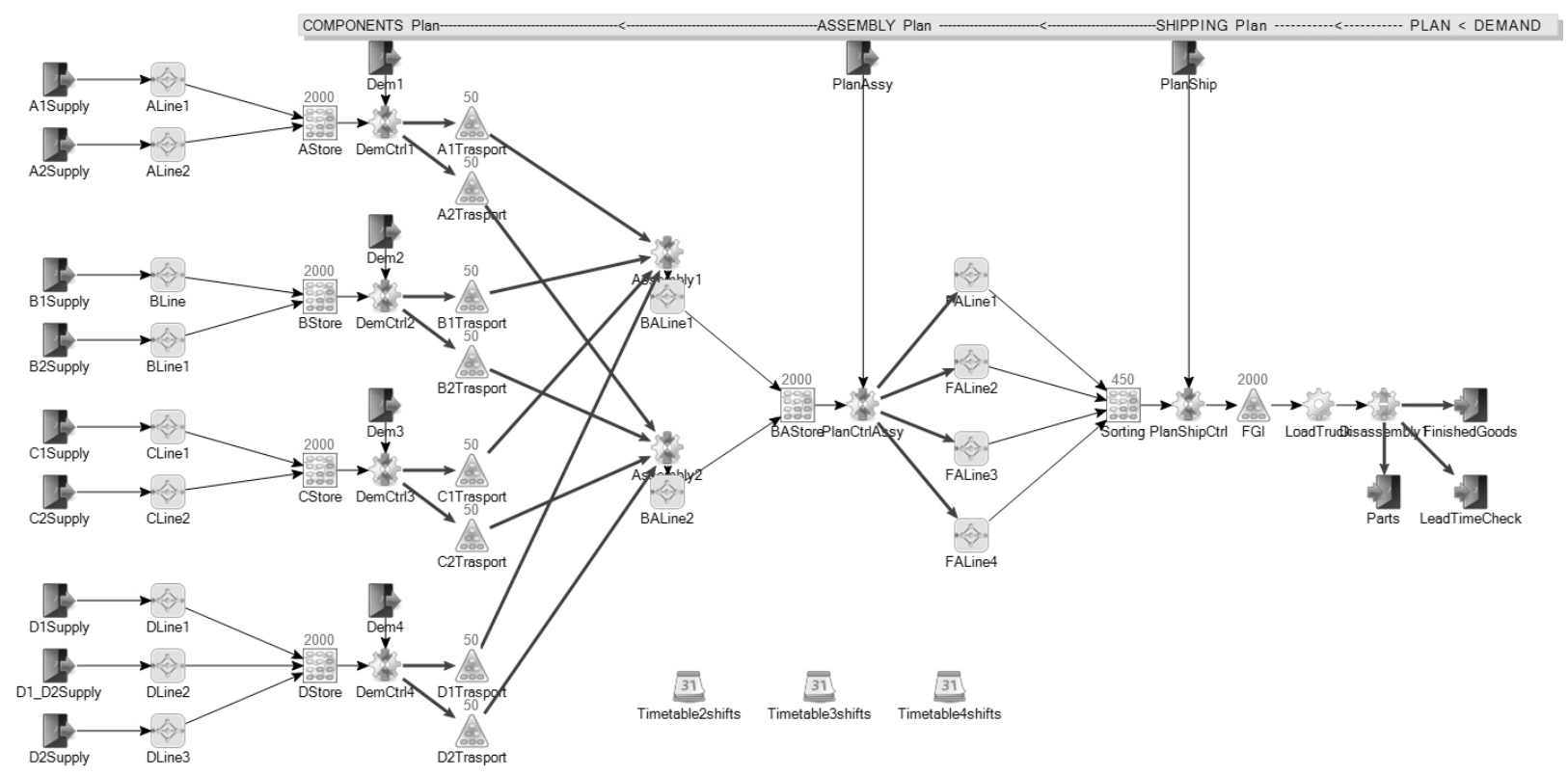

Figure 3: The model used for the application test in FACTS Analyzer.

Stores between the lines are planned in order to enable future addition of variants, altering of the sequence, and to be able to operate various parts of the factory under different shifts. It is especially interesting to be able to run the manual work assembly lines during fewer shifts with lower labor cost through periods of lower customer demand. The scenario described in this paper assumes that the factory 
is running two main product types, or variants, with $50 \%$ of the produced volume each. The conceptual factory was designed to have a throughput of 116 parts per hour with all lines operating simultaneously under these conditions. The nine (automated) machining lines are located first in the sequence, feeding two semi-automated assembly lines through four stores. Components are transported to the assembly lines through automated systems with carriers and conveyors, in this case modeled using buffers. The two automated assembly lines subsequently supply the four assembly lines through a store. After final assembly, the finished products pass through a sorting store and continues to a finish goods inventory (FGI) waiting to be loaded onto trucks in the shipping department. The customer demand is synchronized into the factory in three planning points, of which one is divided into four separate plans connected to the stores after the machining lines. The line objects were modeled as custom objects according to the new aggregation technique proposed in this paper.

\subsection{Simulation Experiments}

The model was prepared with data from the conceptual development project as seen in Table 2. The aggregated lines were individually evaluated in order to verify their respective behavior before implementation in the system model. Input variation was added to some of the aggregated models to enhance their stochastic properties, through input availability and MTTR.

Table 2: Application Test Simulation Model Data.

\begin{tabular}{|l|l|l|l|l|l|l|l|l|}
\hline Line & $\begin{array}{l}\text { Proc } \\
\text { Time (s) }\end{array}$ & Avb. & $\begin{array}{l}\text { MTTR } \\
\text { out } \\
\text { min })\end{array}$ & $\begin{array}{l}\text { WIP } \\
\text { max }\end{array}$ & $\begin{array}{l}\text { WIP } \\
\text { avg }\end{array}$ & $\begin{array}{l}\text { LeadTime } \\
\text { min (min) }\end{array}$ & $\begin{array}{l}\text { Line } \\
\text { Input } \\
\text { Avb. }\end{array}$ & $\begin{array}{l}\text { Line } \\
\text { Input } \\
\text { MTR } \\
\text { (s) }\end{array}$ \\
\hline A1 & 49.66 & 0.8 & 5 & 800 & 500 & 258.6 & 0.95 & 3600 \\
\hline A2 & 49.66 & 0.8 & 5 & 800 & 500 & 258.6 & 0.95 & 3600 \\
\hline B1 & 46.55 & 0.75 & 5 & 1600 & 1000 & 517.2 & 0.9375 & 3600 \\
\hline B2 & 46.55 & 0.75 & 5 & 1600 & 1000 & 517.2 & 0.9375 & 3600 \\
\hline C1 & 43.45 & 0.7 & 5 & 480 & 300 & 155.2 & 0.925 & 3103 \\
\hline C2 & 34.70 & 0.8 & 5 & 480 & 300 & 108.4 & 0.95 & 2169 \\
\hline D1 & 65.45 & 0.8 & 5 & 96 & 60 & 40.9 & 100 & 0 \\
\hline D2 & 65.45 & 0.8 & 5 & 96 & 60 & 40.9 & 100 & 0 \\
\hline D3 & 59.38 & 0.8 & 5 & 96 & 60 & 37.1 & 100 & 0 \\
\hline BA1 & 42.99 & 0.8 & 5 & 128 & 80 & 35.8 & 100 & 0 \\
\hline BA2 & 42.99 & 0.8 & 5 & 128 & 80 & 35.8 & 100 & 0 \\
\hline FA1 & 110.40 & 0.92 & 2 & 45 & 30 & 30.0 & 100 & 0 \\
\hline FA2 & 110.40 & 0.92 & 2 & 45 & 30 & 30.0 & 100 & 0 \\
\hline FA3 & 110.40 & 0.92 & 2 & 45 & 30 & 30.0 & 100 & 0 \\
\hline FA4 & 110.40 & 0.92 & 2 & 45 & 30 & 30.0 & 100 & 0 \\
\hline
\end{tabular}

The capacity of all stores was set to 2000 with $50 \%$ allocated for each of the two main products. All the stores were assumed to have equal processing time, i.e. the minimum time for a product to reside in the store, of one hour. An initial simulation was run, without any shifts configured in the time tables, in order to verify if the model could be considered valid. 


\section{Pehrsson, Frantzén, Aslam, and Ng}

The results were a throughput of 115.35 parts per hour, to be compared with the expected 116 parts per hour and a lead time from 17.3 hours for the fastest product and 25.6 hours for the slowest product, which the company considered to be reasonable under the stated conditions.

\subsection{Optimization}

A very powerful way of testing the aggregation technique and the model was considered to be the application in an optimization study that could provide insights about the resulting characteristics.

An interesting problem to explore was the available trade-offs between throughput (TP), lead time (LT), and lean buffer sum (LB) (Enginarlar, Li, and Meerkov 2005). The same type of study was successfully applied when testing a previously presented aggregation technique (Pehrsson et al. 2014). The problem is basically formulated through the three objectives maximization of $\mathrm{TP}$, minimization of LT, and minimization of LB as shown in Equations 6-8.

$$
\begin{gathered}
f_{1}=\min (\mathrm{LT}) \\
f_{2}=\min \left(\sum_{i=1}^{n} \text { Buffer }_{i_{\text {capacity }}}\right) \\
f_{3}=\max (\mathrm{TP})
\end{gathered}
$$

where $f_{1}$ = objective function for minimization of lead-time, $f_{2}=$ objective function for minimization of the sum of the capacity in Buffer 1 to Buffer $n$, where, $n=$ the number of buffers to be analyzed, and $f_{3}=$ objective function for maximization of throughput.

The input parameters (decision variables) were the capacity of the six stores, within the range [2, 2002], with discrete step of 10 . The task is to dimension the stores in order to enable three shifts operation in the machining lines, FAline2, and FALine4 and two shifts operation in the remaining assembly lines. The capacity will be reduced by the shift restrictions and when running the factory under these conditions, TP is expected to drop down to approximately $60 \mathrm{JPH}$. The optimization was setup with the NSGA-II algorithm (Deb 2001) and was executed through 5000 iterations with 10 replications each. The simulation Horizon was 21 days, including 7 days warm up time.

\subsection{Optimization Results}

The optimization results show an expected behavior with opportunities to make trade-offs between the three objectives as seen in Figure 4. A certain amount of storage capacity is required for the system to deliver high throughput and the required dimensioning of individual storages can be found in the data from the optimization. Too little storage capacity will affect the TP and the LT. Analyses using visual techniques are well suited for knowledge discovery (Weiss and Indurkhya 1998), and also for exploring multi-dimensional data (Simpson and Domnelinger 2009). By plotting each storage capacity, as the example shown in Figure 4, and through a parallel coordinate analysis, shown in Figure 5, the exact level required for each storage capacity to be determined. In order to reach Throughput around $59 \mathrm{JPH}$, a lean buffer capacity of 3332 units, and a lead time close to 33.5 hours, the capacities of the stores should be 472 units for AStore, 682 units for BStore, 512 units for CStore, 842 units for DStore, 562 units for BAStore, and 362 units for the FGI.

\subsection{Findings from the Application Study}

Through the simulation and optimization experiments, it can be concluded that the production system model built with objects based on the proposed new aggregation technique does produce expected results and a natural behavior. 

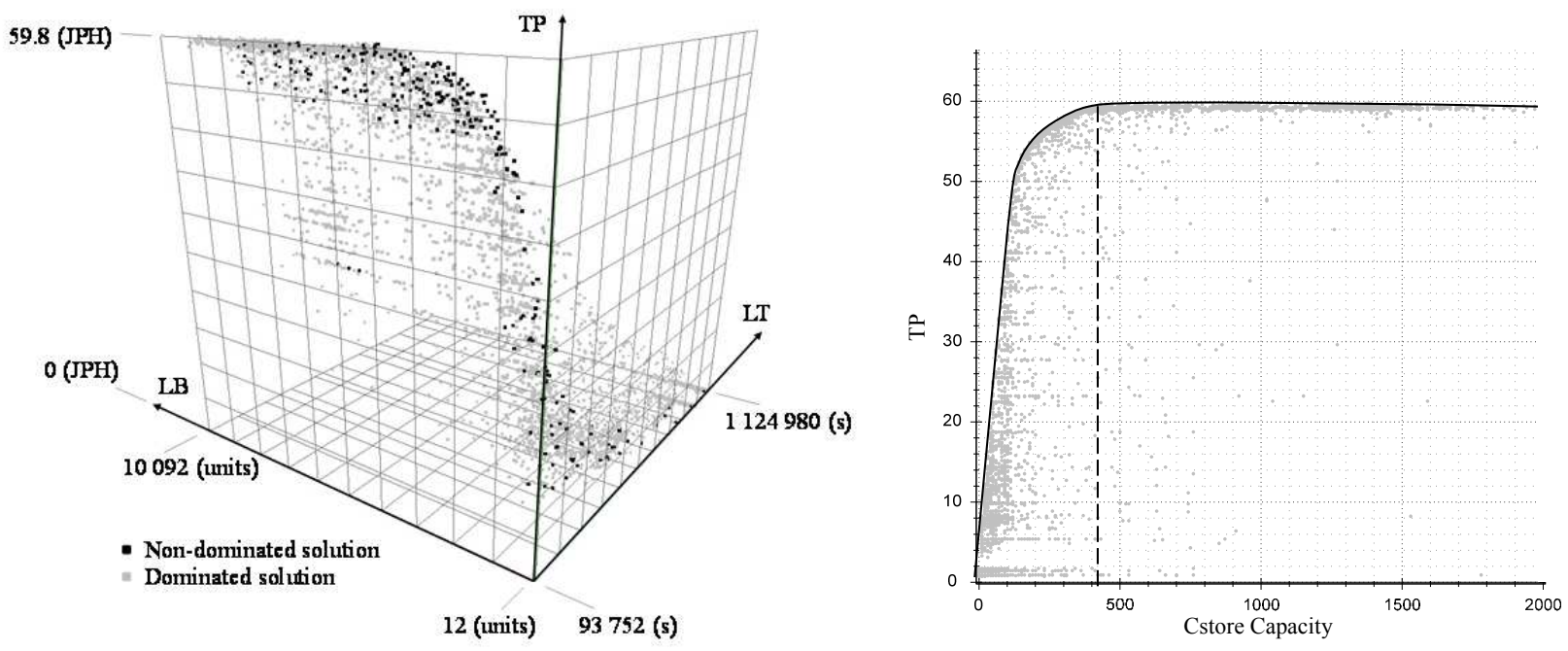

Figure 4: Left: Optimization Results. Right: CStore Capacity Plot with Proposed Solution (dashed).

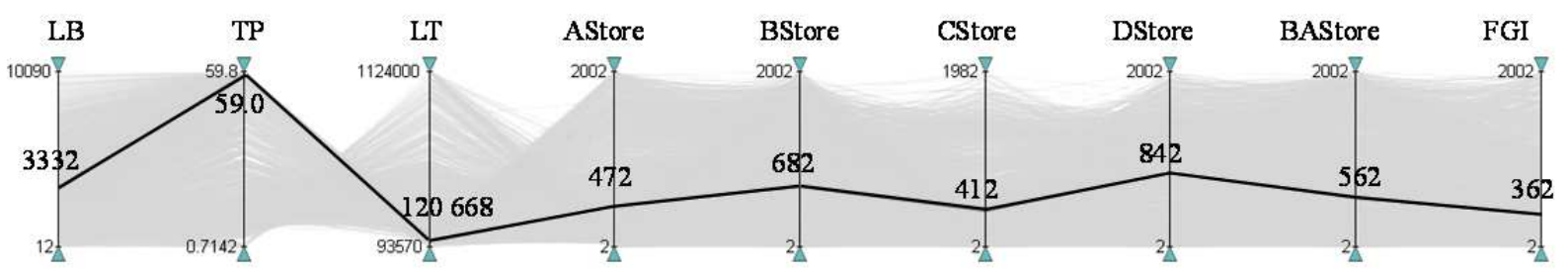

Figure 5: Parallel Coordinate Analysis with the objectives and the proposed Store capacities in black.

The potential opened by the new technique for aggregating line models with DES in order to perform optimizations on conceptual higher level manufacturing systems has been demonstrated and a first proof of concept has been established. Some very useful insights about the manufacturing system were generated through the optimization study. The individual stores in the system can be dimensioned to enable the various lines to be operated on different time schedules while simultaneously taking lead time, the lean buffer sum, and throughput into consideration.

\section{CONCLUSIONS AND FUTURE WORK}

A novel technique for aggregation of DES production line models has been proposed and a first proof of concept has been established. The aggregation technique has successfully been applied on a high level manufacturing system. It can be concluded that such aggregated models can be populated with data using generalized input parameters. The application study shows that the aggregation technique is suitable for conceptual modeling and optimizations. Variation in throughput, lead time and WIP is considered and analyses have shown that the resulting behavior of an aggregated line object is adequate for replicating real world production lines in conceptual studies. However, future work will be required to further validate the modeling technique in terms of variation patterns and subsequently updating the currently used distributions if required. Lines with comparably short lead time or long MTTR will probably require special attention since there might be a risk of statistical interference with inferior line performance as a result. Experiments with lead time dependent, gradually reduced, variation in the WIP control loop could be one way of handling such issues. In the application study only two main variants was included. The analysis of manufacturing systems with many variants that could truly reflect the potential of the proposed technique should be included in future studies. 
Pehrsson, Frantzén, Aslam, and Ng

\section{REFERENCES}

Aslam, T. 2013. "Analysis of Manufacturing Supply Chains Using System Dynamics and MultiObjective Optimization", PhD Dissertation, University of Skövde, Skövde, Sweden (ISBN 97891981474-1-4)

Aslam, T., P. Hedenstierna, A.H.C. Ng., L. Wang, 2011. "Multi-Objective Optimisation in Manufacturing Supply Chain Systems Design: A Comprehensive Survey and New Directions", In Multi-Objective Evolutionary Optimisation for Product Design and Manufacturing, edited by L. Wang, A.H.C. Ng, K. Deb , pp. 35-70, Springer London.

Banks, J., J. S. Carson, B. L. Nelson, and D. M. Nicol. 2010. Discrete-Event System Simulation. 5th ed. Upper Saddle River, New Jersey: Prentice-Hall, Inc.

Beamon, B.M, 1998, "Supply Chain Design and Analysis: Models and Methods." Int. J. Production Economics. 55, 281-294.

Chwif, L., M.R.P. Barretto, and R.J. Paul. 2000. "On Simulation Model Complexity." In: Joines JA, Barton RR, Kang K and Fishwick PA (eds). In Proceedings of the 2000 Winter Simulation Conference. Piscataway, New Jersey: Institute of Electrical and Electronics Engineers, Inc. pp. 449455.

Curry, G.L., and R.M. Feldman. 2011. Manufacturing Systems Modeling and Analysis, $2^{\text {nd }}$ edition, Springer-Verlag Berlin Heidelberg.

Deb, K. 2001. Multi-objective optimization using evolutionary algorithms, 3'rd ed., Wiley, Wiltshire, $\mathrm{UK}$.

Deb, K., and A. Srinivasan. 2006. "Innovization: Innovating Design Principles Through Optimization." In Proceedings of the Genetic and evolutionary Computation Conference (GECCO-2006), The Association of Computing Machinery (ACM), New York, 1629-1636.

Dudas, C., A.H.C. Ng, L. Pehrsson, and H. Boström. 2013. "Integration of Data Mining and MultiObjective Optimization for Decision Support in Production System Development." International Journal of Computer Integrated Manufacturing. DOI:10.1080/0951192X.2013.834481

Duffie, N.A. and L. Shi. 2009. "Maintaining Constant WIP-Regulation Dynamics in Production Networks with Autonomous Work Systems." CIRP Annals - Manufacturing Technology, 58, 399-402.

Enginarlar, E., J. Li and S.M. Meerkov. 2005. "How Lean Can Lean Buffers Be?" IIE Transactions, 37, 333-342.

Frantz, F. K. 1995. "A Taxonomy of Model Abstraction Techniques." In: Alexopoulos C, Kang K, Lilegdon W R, and Goldsman D (eds.). In Proceedings of the 1995 Winter Simulation Conference, Piscataway, New Jersey: Institute of Electrical and Electronics Engineers, Inc. pp. 1413-1420.

Hopp, W.J. and M.L. Spearman. 2000. Factory physics: foundations of manufacturing management, 2nd ed. Irwin McGraw-Hill Higher Education, Burr Ridge, IL.

Hung, W.Y., N.J Samsatli, N Shah. 2006. "Object-Oriented Dynamic Supply-Chain Modelling Incorporated with Production Scheduling.” European J. of Operational Research, 169, 1064-1076.

Little, J. 1961. "A Proof of the Queueing Formula L= $\lambda$ W." In Operations Research 9:383-387, 1961.

Madan, M., Y.-J. Son, H. Cho, B. Kulvatunyou. 2005. "Determination of Efficient Simulation Model Fidelity for Flexible Manufacturing Systems." International Journal of Computer Integrated Manufacturing. Vol. 18, No. 2-3, pp. 236-250.

Morecroft, J. 2007. Strategic Modelling and Business Dynamics: A Feedback Systems Approach. John Wiley \& Sons.

Ng, A.H.C., C. Dudas, L. Pehrsson, and K. Deb. 2012. "Knowledge Discovery in Production Simulation by Interleaving Multi-Objective Optimization and Data Mining." In Proceedings of the 5'th Swedish Production Symposium (SPS'12), Linköping, Sweden, 6-8 November 2012.

Ng, A.H.C., J Bernedixen, M. Urenda Moris, M Jägstam. 2011. "Factory Flow Design and Analysis Using Internet-Enabled Simulation-Based Optimization and Automatic Model Generation.” In: Jain 
S, Creasey R R, Himmelspach J, White K P, and Fu M (eds.). In Proceedings of the 2011 Winter Simulation Conference, Piscataway, New Jersey: Institute of Electrical and Electronics Engineers, Inc, pp. 2176-2188.

Pehrsson, L., S. Lidberg, M. Frantzén, T. Aslam, and A.H.C. Ng. 2014. "Aggregated Discrete Event Modelling for Simulation and Optimisation of Manufacturing Systems." In Proceedings of the Industrial Simulation Conference, ISC'14, Skövde, June 11-13, 2014, Eurosis. 83-90.

Pehrsson, L. and A.H.C. Ng. 2011. "An Applied Framework for Simulation-Based Multi-Objective Production System Optimisation Within Production System Development." In Proceedings of the Industrial Simulation Conference, ISC'2011, June 6-8, 2011, Venice, Italy

Rooda., J.E. and J. Vervoort, 2007. "Analysis of Manufacturing Systems Using $x$ 1.0. Eindhoven University of Technology." source: http://seweb.se.wtb.tue.nl/education/mis/AMS_main.pdf, Accessed March 2014.

Simpson, T.W. and J.A. Donndelinger. 2009. "Visual Steering Commands for Trade Space Exploration: User-Guided Sampling With Example." Journal of Computing and Information Science in Engineering. Vol. 9.

Weiss S.M. and N. Indurkhya. 1998. Predictive Data Mining: a practical guide. Morgan Kaufmann Publishers, Inc., San Fransisco.

\section{AUTHOR BIOGRAPHIES}

LEIF PEHRSSON is a Senior Manager, Research and Technology Development, Engine Manufacturing Engineering, at Volvo Car Group and an Affiliated Senior Lecturer at the University of Skövde, Sweden. He holds a Ph.D. degree in Manufacturing Systems from De Montfort University in the UK., a M.Sc. degree in Automation Engineering, and a B.Sc. degree in Mechanical Engineering. He has been working in various engineering and managerial positions within automotive industry for 20 years. His e-mail address is leif.pehrsson@his.se.

MARCUS FRANTZÉN holds a Ph.D. degree in Manufacturing Engineering from De Montfort University in the UK. He has a M.Sc. degree in Manufacturing Management from the Loughborough University and a B.Sc. degree in Automation Engineering from the University of Skövde, Sweden. He has more than ten years' experience from industrial simulation modelling and experimentation. Currently, he is an Assistant Professor at the University of Skövde. His e-mail address is marcus.frantzen@his.se.

TEHSEEN ASLAM holds a Ph.D. in industrial informatics from University of Skövde, Sweden. He has a M.Sc. degree in Manufacturing Management from Loughborough University and a B.Sc. in Automation Engineering from University of Skövde. His research interests include modelling, simulation and multiobjective optimisation for the design and analysis of supply chains. His e-mail address is tehseen.aslam@his.se.

AMOS H.C. NG is a Professor of Production and Automation Engineering at the University of Skövde, Sweden. He holds a Ph.D. degree in Computing Sciences and Engineering. His main research interest lies in applying multi-objective optimization and data mining techniques for production systems design, analysis and improvement. His e-mail address is amos.ng@his.se. 